\title{
Optimizing Electrospray Interfaces Using Slowly Diverging Conical Duct (ConDuct) Electrodes
}

\author{
Andrew N. Krutchinsky, Júlio C. Padovan, Herbert Cohen, Brian T. Chait
}

Laboratory for Mass Spectrometry and Gaseous Ion Chemistry, The Rockefeller University, New York, NY 10065, USA

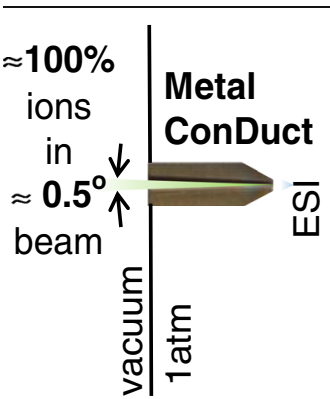

Abstract. We demonstrate that the efficiency of ion transmission from atmosphere to vacuum through stainless steel electrodes that contain slowly divergent conical duct (ConDuct) channels can be close to $100 \%$. Here, we explore the properties of $2.5-\mathrm{cm}$ long electrodes with angles of divergence of $0^{\circ}, 1^{\circ}, 2^{\circ}, 3^{\circ}, 5^{\circ}, 8^{\circ}, 13^{\circ}$, and $21^{\circ}$, respectively. The ion transmission efficiency was observed to jump from 10-20\% for the $0^{\circ}$ (straight) channels to $90-95 \%$ for channels with an angle of divergence as small as $1^{\circ}$. Furthermore, the $2-3^{\circ}$ ConDuct electrodes produced extraordinarily low divergence ion beams that propagated in a laser-like fashion over long distances in vacuum. To take advantage of these newly discovered properties, we constructed a novel atmosphere-to-vacuum ion interface utilizing a $2^{\circ}$ ConDuct as an inlet electrode and compared its ion transmission efficiency with that of the interface used in the commercial (Thermo Fisher Scientific, San Jose, CA, USA) Velos Orbitrap and Q Exactive mass spectrometers. We observed that the ConDuct interface transmitted up to 17 times more ions than the commercial reference interface and also yielded improved signal-to-noise mass spectra of peptides. We infer from these results that the performance of many current atmosphere-to-vacuum interfaces utilizing metal capillaries can be substantially improved by replacing them with $1^{\circ}$ or $2^{\circ}$ metal ConDuct electrodes, which should preserve the convenience of supplying ion desolvation energy by heating the electrode while greatly increasing the efficiency of ion transmission into the mass spectrometer.

Keywords: Electrospray ionization, ESI, Ambient atmosphere to vacuum interface, ESI interface, Slowly divergent conical duct, ConDuct, High efficiency ion transmission, $100 \%$ efficient ion transmission, Sharply collimated ion beams, Low divergence ion beams, Metal ConDucts

Received: 7 July 2014/Revised: 2 December 2014/Accepted: 3 December 2014/Published Online: 10 February 2015

\section{Introduction}

$\mathrm{M}$ ass spectrometry (MS) relies on the production and manipulation of ion beams to deliver ions to mass analyzers [1-7]. A typical electrospray ionization (ESI) MS experiment starts with sample ionization under ambient conditions in the laboratory atmosphere followed by entrainment of the resulting ions into a carrier gas that expands through some type of transfer conduit into the vacuum, where the ions are manipulated and analyzed. The efficiency of such ESI-MS analysis strongly depends on the overall efficiency of the ion delivery system, which in turn depends on efficiencies of several interrelated processes, such as sample ionization, ion desolvation,

Electronic supplementary material The online version of this article (doi:10.1007/s13361-014-1063-0) contains supplementary material, which is available to authorized users.

Correspondence to: Brian Chait; e-mail: chait@rockefeller.edu ion transfer through the atmosphere-to-vacuum interface, and ion guidance into the mass analyzer. Although several recently introduced techniques have significantly improved the efficiency of sample ionization [8-12] and ion guidance in vacuum [13-15], there remains much room for improving the efficiency of ion transfer from atmosphere into the vacuum, a process that continues to rely mainly on the use of two types of inlet.

The first type consists of a simple orifice at the tip of a diverging or converging "cone" electrode, usually with open angles in the range $10-340^{\circ}[1,16-18]$. A thin, flat electrode with a small orifice can be viewed as a special case of this type of electrode with an open angle of $180^{\circ}$. In this first type of electrode, the flow of gas sucked into the vacuum through the orifice has a very limited time to interact with its walls, separating from the orifice boundary within microseconds [19, 20]. Because of the brevity of this interaction, the efficiency of ion transfer by gas flow through the electrode can approach $100 \%$ [21]. In addition, because the flow separates at the orifice, the open angle of the "cone" electrode plays little role in the 
formation of the beam (at least for the range of open angles indicated above [16, 22, 23]). Despite their high transmission efficiency, such orifice-based ion interfaces are not efficient in supplying energy for desolvation of ESI droplets that do not undergo full evaporation or ions that remain solvated during gas expansion into the vacuum [21].

The second type of widely used atmosphere-to-vacuum inlet consists of long, metal or glass capillaries [24-26]. In contrast to orifice-based inlets, the flow through such capillaries can take milliseconds. The resulting two to three orders of magnitude longer interaction time with the boundaries of the inlet electrode render the ion desolvation process more efficient and easier to implement by simply heating the capillary to an appropriately selected temperature, usually in the range 100 $300^{\circ} \mathrm{C}$. The biggest drawback of such long capillary inlet electrodes is their suboptimal ion transmission efficiencies, which are usually in the range $1-20 \%$ depending on their particular geometry as well as details related to the ESI spray emitter [6, 21].

We recently discovered that a slightly divergent inlet channel can boost the ion transmission efficiency to almost $100 \%$ [21]. Furthermore, this type of channel creates an ion beam that diverges in an extraordinarily slow way in a "laser-like" fashion over long distances in vacuum. In our first implementation of such an electrode, which we termed "ConDuct", we used a conductive plastic pipette tip containing a 7-mm-long, $1.6^{\circ}$ divergent channel having an entrance diameter of $0.4 \mathrm{~mm}$ [21]. Although large angle converging/diverging cones have been extensively employed in the formation of gas beams $[1$, 16-18, 20], the improved ESI ion transmission via flow laminarization [27] at such small angles has not previously been observed experimentally.

To examine this phenomenon in more detail, we investigated the properties of ion beams created by a series of conical channels, determining ion transmission and beam properties as a function of channel divergence angle. To do this, we produced a series of 24-mm-long stainless steel ConDuct electrodes with angles of $0^{\circ}, 1^{\circ}, 2^{\circ}, 3^{\circ}, 5^{\circ}, 8^{\circ}, 13^{\circ}$, and $21^{\circ}$, respectively. We also describe an atmosphere-to-vacuum ion interface that utilizes ConDuct electrodes as an inlet to a mass spectrometer, a "ConDuct interface", and developed a technique for quantitative assessment of its performance relative to that of widely used commercially available interfaces.

\section{Experimental}

Most of the experimental apparatus used in this work are described in detail in [21].

\section{Metal ConDuct Electrodes}

Eight 25.4-mm-long ConDuct electrodes with channel divergence following the Fibonacci series [29], $0^{\circ}, 1^{\circ}, 2^{\circ}, 3^{\circ}, 5^{\circ}, 8^{\circ}$, $13^{\circ}$, and $21^{\circ}$, respectively, were produced by Midwest Precision Tool and Die, Inc. (Sioux Falls, SD, USA) from 440-grade stainless steel using electrical discharge machining (EDM) [28]. One of these ConDuct electrodes (channel divergence $2^{\circ}$ ) was cut in half to expose its cross-section (Figure 1a).

\section{Measurements of the ESI Ion Current Transmission Efficiency}

The apparatus used to measure the efficiency of ion transmission into the vacuum (Figure $1 \mathrm{~b}$ ) consisted of a vacuum chamber evacuated to a few Torr; a nanospray ion source facing the inlet of a ConDuct electrode supported by a holder, which could be heated to a few hundred degrees centigrade; and a Faraday cup, which intercepted the ion beam 10-160 $\mathrm{mm}$ downstream of the ConDuct electrode exit. A picoammeter (model 480; Keithley Instruments, Inc., Cleveland, OH, USA) was connected to either the Faraday cup to measure the transmitted ion current or the ConDuct electrode to measure transmission losses. The total ESI ion current was measured by simultaneously connecting both electrodes to the picoammeter.

\section{Measurements of the Diameter of the Beam}

The setup for measurements of the diameter of the beams created by the ConDuct electrodes with different channel divergences is shown in Figure 2a. We used the same apparatus as that used for measurements of the ion transmission efficiency (above), except that the Faraday cap was replaced by a holder for replaceable targets, cut from 1-mm-thick sheets of conductive polystyrene (GoodFellow Corp., Coraopolis, PA, USA) to dimensions of $2.54 \mathrm{~cm}$ by $2.54 \mathrm{~cm}$. These targets intercepted ion beams that contained SYPRO Ruby (Molecular Probes, Life Technologies, Grand Island, NY, USA), a fluorescent dye, electrosprayed at the inlets of the different ConDuct electrodes. The distance between the targets and the exit from the ConDuct electrode was set to $80 \mathrm{~mm}$ and the target exposure time to the beam was usually $3-30 \mathrm{~min}$. The deposited spots of dye were visualized using a Typhoon 9410 molecular imager (GE Amersham Molecular Dynamics, Ramsey, MN, USA).

\section{Visualizing the Ion Beam}

A schematic of the experimental setup constructed for visualizing the ion beam is shown in Figure 4a. It consists of an ESI source with a microspray or nanospray emitter positioned close to the inlet electrode mounted on one flange of a 500-mm-long vacuum chamber made of square aluminum pipe. The opposite flange contained an acrylic window through which we shone a laser beam towards the gas/ion beam that originated at the other end of the pipe. The diameter of the laser beam could be controlled up to $1.5 \mathrm{~cm}$ via a system of telescopic lenses. The light scattered by particulates present within the gas/ion beam was observed in the dark through slit-shaped windows built on the sides of the vacuum chamber. We used a $10-\mathrm{mW}$ diodepumped solid state green laser (532 nm, DJ532-10; Thor Labs, 

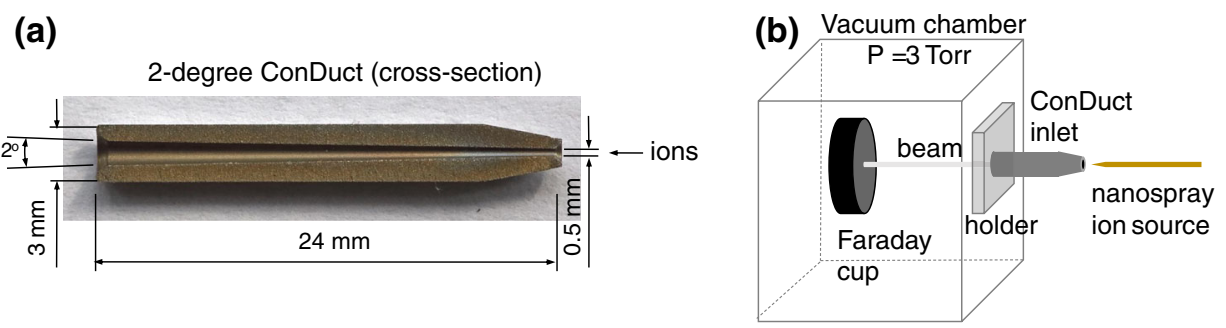

(c)

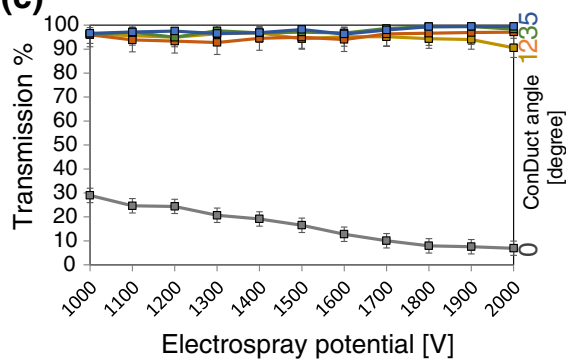

(d)

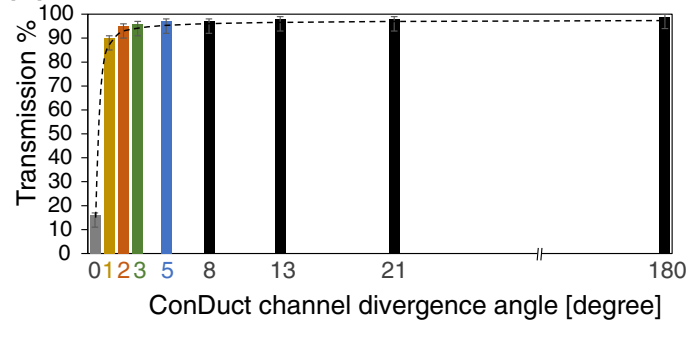

Figure 1. Measurements of the ion transmission efficiencies of a series of metal ConDuct electrodes. (a) Cross-section of a stainless steel ConDuct electrode with a $2^{\circ}$ divergent channel showing its major dimensions. (b) Experimental setup for measurement of ion transmission efficiency. ConDuct electrodes with different angles of channel divergence can be rapidly exchanged from the heatable electrode holder. (c) Transmission efficiency of the metal conduct electrodes versus ESI potentials for inner channel divergence angles of $0^{\circ}, 1^{\circ}, 2^{\circ}, 3^{\circ}$, and $5^{\circ}$, respectively. (d) Average transmission efficiency of different ConDuct electrodes for ESI voltages in the range 1000-2000 V. A flat, thin electrode having an orifice diameter of $0.5 \mathrm{~mm}$ can be considered a special case of the ConDuct electrode with an angle of divergence of $180^{\circ}$. The dashed line indicates a significant increase in transmission efficiency when the angle of divergence is increased from $0^{\circ}$ to $1^{\circ}$.

Newton, NJ, USA), powered by an in-house designed and constructed power supply, which generates square pulses of 3$4 \mathrm{~V}$ amplitude at a frequency of $200 \mathrm{kHz}$. The vacuum chamber was evacuated by a single Edwards 12 rotary pump (Edwards Vacuum, Albany, NY, USA) to a pressure of a few Torr.

\section{Measurements of the Relative Ion Transmission Efficiencies of the Two Atmosphere-to-Vacuum Interfaces}

Figure 5a shows a schematic of a modified LCQ DECA XP mass spectrometer equipped with two atmosphere-to-vacuum (a)

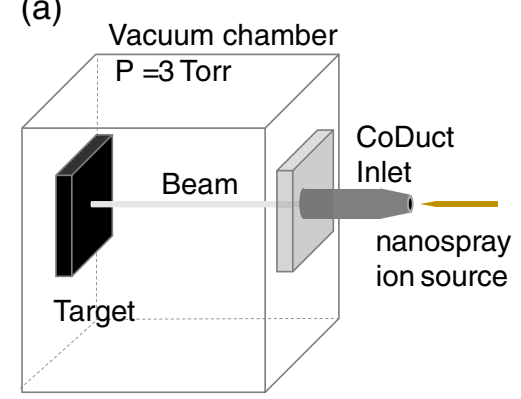

(b) (c)

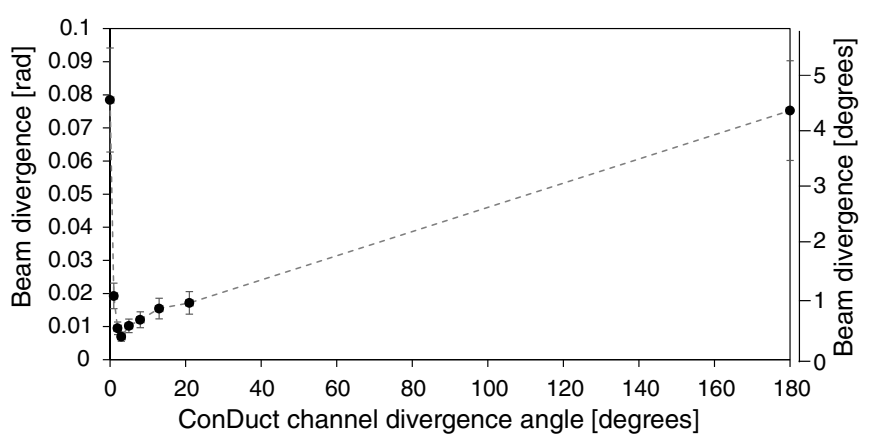

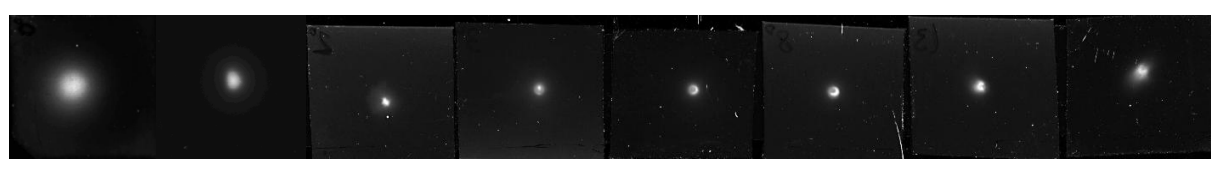

$0^{\circ} \quad 1^{\circ}$ $2^{\circ}$ $3^{\circ}$ $5^{\circ}$ $8^{\circ}$ $13^{\circ}$

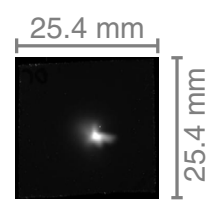

$180^{\circ}$

Figure 2. Effective diameter of the beam produced by ConDuct electrodes with different angles of channel divergence. (a) The experimental set up used for measurements of beam diameter. (b) Images of the spots of fluorescent SYPRO Ruby dye deposited on conductive plastic targets. The targets were exposed to the beams created by a series of different ConDuct electrodes. (c) Angular distribution of SYPRO Ruby dye of the beams produced by the ConDuct electrodes with different channel divergence angles. A straight capillary and a flat thin electrode with an orifice can be considered as special cases having divergence angles of $0^{\circ}$ and $180^{\circ}$, respectively. 
interfaces [21]. The two interfaces can accept ions from two ESI ion sources simultaneously and transmit them along two arms of a T-quadrupole $q_{T}[13,21,30]$, wherein ions are combined and transferred through an aperture in electrode $e_{1}$ and quadrupole ion guide $q_{1}$ towards the ion trap mass analyzer. The detailed description of the instrument can be found in in a companion paper [21] and in the Supplementary Material.

\section{Sample Preparation}

For the measurements of ion current, we utilized a solvent mixture consisting of $60 \% \mathrm{v}$ methanol and $1 \% \mathrm{v}$ acetic acid in water. For measurements of the diameter of the beam, we used a solution containing the fluorescent dye SYPRO Ruby as well as a solution of 5-nm average-size nanogold particles (Nanoprobes, Yaphank, NY, USA, cat. 2010, $30 \mathrm{nmol}$ ). See Supplemental Information for details.

We used four peptides to measure the relative ion transmission efficiency. These peptides were made by AnaSpec, Inc. (Fremont, CA, USA) and consisted of unlabeled angiotensin I (cat. 20627, sequence DRVYIHPFHL, monoisotopic MM $=1295.7 \mathrm{u}$ ), labeled angiotensin I (cat. 65140 , sequence $\operatorname{DRV}\left(5^{13} \mathrm{C}\right.$ $\left.{ }^{15} \mathrm{~N}\right) \mathrm{YI}\left(6{ }^{13} \mathrm{C}^{15} \mathrm{~N}\right) \mathrm{HPFHL}$, monoisotopic $\left.\mathrm{MM}=1308.7 \mathrm{u}\right)$, unlabeled $\beta$-amyloid peptide, fragment 1-15 (cat. 61798, sequence DAEFRHDSGYEVHHQ, monoisotopic MM $=1825.8 \mathrm{u}$ ), and labeled $\beta$-amyloid peptide fragment $1-15$ (cat. 61798, sequence $\operatorname{DA}\left(3^{13} \mathrm{C}^{15} \mathrm{~N}\right) \operatorname{EFR}\left(6^{13} \mathrm{C} 4^{15} \mathrm{~N}\right) \operatorname{HDSG}\left(213 \mathrm{C}{ }^{15} \mathrm{~N}\right)$ YEVHHQ, monoisotopic $\mathrm{MM}=1842.8 \mathrm{u}$ ). We prepared two equimolar peptide solutions by mixing the unlabeled and the labeled forms of each peptide to a final concentration of $100 \mathrm{fmol} / \mu \mathrm{L}$ each in $60 \% \mathrm{v}$ methanol, $1 \% \mathrm{v}$ acetic acid.

\section{Results and Discussion}

\section{Transmission of the ESI Current from Atmosphere into Vacuum}

We measured the efficiency of ion transmission through the set of manufactured ConDuct electrodes using the setup shown in Figure $1 \mathrm{~b}$. Here, we electrosprayed a blank solution of watermethanol-acetic acid at 39:60:1 (v/v/v) delivered at a flow rate of $400 \mathrm{~nL} / \mathrm{min}$ to the tip of the $15-\mu \mathrm{m}$ nanospray emitter positioned $1 \mathrm{~mm}$ from the inlets of the ConDuct electrodes. Figure 1c provides the measured transmission efficiencies of the $0^{\circ}, 1^{\circ}, 2^{\circ}, 3^{\circ}$, and $5^{\circ}$ ConDuct electrodes, defined as the ratio between the transmitted current and the total ESI current for ESI voltages between 1000 and $2000 \mathrm{~V}$. Strikingly, the transmission efficiency was $90-100 \%$ for all tested ConDuct electrodes with divergences equal to or greater than $1^{\circ}$, whereas it was only $10-30 \%$ for the zero-degree "ConDuct" electrode. Figure $1 \mathrm{~d}$ reveals how the transmission efficiency, averaged over ESI voltages from 1000 to $2000 \mathrm{~V}$, depends on the channel divergence angle for all the ConDuct electrodes, including a $180^{\circ}$ "ConDuct" electrode, which is just a flat, thin electrode with an orifice diameter of $0.5 \mathrm{~mm}$. Assuming a smooth behavior between the data points, we observed a sudden jump in average ion transmission efficiency from 10$20 \%$ for the 0 -degree, straight channel to $90-95 \%$ for channels with angles of divergence as small as $1^{\circ}$. These results raise the question as to why the transmission efficiency increases so drastically as soon as the divergence angle rises slightly above zero.

Our hypothesis is that the throat of the gas flow [1,22], which for the straight capillary is situated right at its exit, suddenly relocates towards its entrance, for channel divergences as small as $1^{\circ}$, in a manner similar to a phase transition. This process is accompanied by laminarization of the flow [27], which leads to minimization of the interactions between the ions embedded in the gas flow and the channel boundaries. For the $0^{\circ}$, straight channel, the gas flow separates from the walls of the capillary at its exit [22], and until then the ions carried by the gas can be lost to the walls of the capillary, especially when the flow is not fully laminar [21,31]. As the angle of divergence increases, the gas flow likely separates from the inner walls of the divergent channel $[22,23]$ at progressively smaller distances from the entrance of the ConDuct electrode, and for the extreme case of the $180^{\circ}$ channel (i.e., a small orifice in a flat electrode), the flow separates right at the orifice. Our next experiments were designed to test this hypothesis.

\section{Measurement of the Width of the Beams Created by Different ConDuct Electrodes}

In our previous work, we noticed that the ConDuct electrode made out of a conductive plastic pipette tip creates a very narrow ion beam, with a divergence of less than $1^{\circ}$ (full width at half maximum) [21]. The low divergence of this ion beam is in sharp contrast to the divergence of the beams created by the orifice and the straight metal capillary, which both diverge by approximately $7^{\circ}[21]$. Furthermore, the beam created by the ConDuct electrode propagates for a long distance without significant dispersion. Indeed, to define the radial distribution of ions in the beam with good spatial resolution, it proved to be necessary to move the $0.5-\mathrm{mm}$ diameter wire probe at least $90 \mathrm{~mm}$ downstream from the exit of the ConDuct electrode. Conversely, to profile the radial distribution of the ions in the beams produced by the orifice and the capillary, it proved to be necessary to move the probe much closer to these two electrodes because the gas flow through them adopts a short, barrel shaped structure, which quickly disappears downstream from the Mach disc [32].

The beams created by some of the ConDuct electrodes were so sharp and focused that it proved to be challenging to accurately determine the radial distribution with the $0.5 \mathrm{~mm}$ wire. Thus, we also implemented a technique that relies on measurements of the diameter of the spot left on targets that were exposed for some time to beams containing the florescent dye SYPRO Ruby [33] (Figure 2a). Figure 2b shows images of the fluorescence spots of dye deposited on these targets exposed to the beams produced by the different inlet electrodes. The beam exposure time and the interception distance were varied as needed to ensure a readily visible fluorescent deposit. In the cases of beams generated by flow through straight channels or orifices in flat electrodes (i.e., $0^{\circ}$ and $180^{\circ}$ divergence angles, 
respectively), the target exposure time was 20-30 min and the distance was $35 \mathrm{~mm}$. Because the beams produced by the ConDuct electrodes with divergence angles greater than or equal to $1^{\circ}$ were much sharper, the exposure time was shortened to 3-5 min and the distance was increased to $80 \mathrm{~mm}$. The images of the spots were obtained with a spatial resolution of $0.1 \mathrm{~mm}$. Using ImageJ analysis software [34], we determined the effective diameter of each spot containing $90-95 \%$ of the deposited dye, and then divided the resulting value by the distance to the target in order to obtain the full beam divergence angle. Figure $2 \mathrm{c}$ shows this beam divergence as a function of the channel divergence angle of the ConDuct electrodes. As before, we assumed a smooth behavior between the data points.

The plot reveals a sudden decrease in the diameter of the beam when the angle of divergence is above zero. Thus, the $1^{\circ}$ ConDuct electrode produced a beam with a divergence of $(1.15 \pm 0.25)^{\circ}$, similar to the divergence of the channel itself, indicating that the flow of gas likely did not separate appreciably from the walls of the electrode all the way to its exit. The ConDuct electrodes with divergence angles of $2^{\circ}, 3^{\circ}$, and $5^{\circ}$ produced the sharpest beams with angular divergences in the range $0.3-0.6^{\circ}(0.005-0.01 \mathrm{rad})$, implying that the flow through the electrodes likely separates from the inner walls of the divergent channel at some distance prior to reaching their exits [22]. After passing this minimum divergence, the diameter of the beams slowly increased as a function of increased channel divergence all the way to $180^{\circ}$, again assuming a smooth behavior between the data points (Figure 2c).

Close examination of the targets exposed to the beams produced by the 2-, 3-, and 5-degree ConDuct electrodes in fluorescent light revealed a small dark mark slightly off-center of each fluorescent spot. These dark marks were also visible in ambient light, appearing like tiny "burnt" holes on the surface. After wiping the target with methanol, we were unable to completely remove these marks, which indicated that the surface was damaged to some extent. We hypothesized that the markings were produced by heavy clusters enriched towards the central line of the beam, arising from focusing phenomena in these high flux gas beams [1].

To test this hypothesis, we electrosprayed a $1 \mu \mathrm{M}$ solution of 5-nm gold particles at the inlet of a 3-degree ConDuct electrode and intercepted the resulting beam with a $1-\mu \mathrm{m}$ nickel foil positioned $80 \mathrm{~mm}$ downstream of the ConDuct exit. After a 30-min exposure, we observed that the beam ablated a small hole through the nickel foil. Figure 3 shows the target and light passing through this small hole. It also shows a 0.3 to $0.4-\mathrm{mm}$ diameter crater on the surface of the foil surrounding the hole. An estimate of the energy needed to ablate such a hole was in agreement with the total energy deposited by impacts of gold clusters focused to approximately 3 mrad. Remarkably, this observed ablation was achieved without application of any voltage to the beam.

\section{Visualizing the Beam}

Figure 4a shows the experimental setup that we built to investigate the properties of the beams created by the ConDuct electrodes. We introduced a 50 -cm-long vacuum chamber with slit-like windows for beam observation and included a pulsed 532-nm laser for illuminating the gaseous ionic beam. We noticed that the ion/gas beam could be readily observed because it contained small droplets of the ESI solution that were not fully evaporated, which scattered the light of the incoming laser beam. To ensure that we observed the true radial distribution of the ion beam itself and not the laser beam, we expanded the diameter of the laser beam to $1.5 \mathrm{~cm}$. Figure $4 \mathrm{~b}$ shows a $15-\mathrm{cm}$-long section of the gaseous beam produced by the 3 -degree ConDuct electrode. The ion beam is laser-like tight and its diameter remained less than $1 \mathrm{~mm}$ along the path shown. Its diameter increased slightly to approximately 2-3 mm further downstream towards the rear window at a distance of $50 \mathrm{~cm}$ from the exit of the ConDuct electrode. The beam stopped being visible when the ESI was switched off (Figure 4c) or when the tip of the ESI emitter was moved away from the inlet of the ConDuct electrode to a distance of 4-7 mm. This observation provides an important clue as to why our best signal-to-noise spectra were observed when the ESI emitter tip was moved away from the inlet and slightly off axis [21]. Apparently, complete evaporation of the electrosprayed droplets could not be achieved when the tip of the emitter was positioned very close $(1-3 \mathrm{~mm})$ to the inlet of short, 24-mm-long ConDuct electrodes even when the temperature of this electrode was elevated. Thus, the task of obtaining the highest yield of ions that are useful for MS analysis still requires a compromise between the emitter-inlet distance, the velocity of the gas sucked at the inlet [21], the ESI voltage, and the temperature and length of the inlet electrode.

When we probed the gaseous ionic beam with a more highly focused pulsed laser beam, and took multiple photographs of the same region, we noticed that sometimes we could detect traces of single particles. We hypothesized that when the intersection volume between the ion beam and the laser beam is sufficiently small, we can sometimes illuminate just a single particle if it happened to be carried by the gas flow through that region. Thus, we took multiple photographs of such gas beams scattering light from this narrow, sub-millimeter diameter laser beam in the region 30-35 cm downstream of the ConDuct exit. Then we analyzed these images to search for characteristic patterns of periodic light traces that should be left by single particles scattering laser light modulated at a frequency of $200 \mathrm{kHz}$. Figure 4d shows one such pattern. We counted approximately six light streaks over a distance of $10 \mathrm{~mm}$ resulting in an average distance of $(1.6 \pm 0.2) \mathrm{mm}$ traveled by the particle in the 5- $\mu$ s duration between pulses. This measurement yielded an estimate for the velocity of the particles in the beam of (320 \pm 40) $\mathrm{m} / \mathrm{s}$. This result indicates that not only does the gas beam disperse very little, even $30-35 \mathrm{~cm}$ downstream of the ConDuct exit, but also that the velocity of the beam is close to or perhaps even slightly higher than the speed of sound.

\section{Comparing the Performances of the Two-degree ConDuct-Based Interface with the Commercial Thermo S-Lens Interface}

Because we deduced that separation from the electrode boundaries occurs relatively early within the $5-21^{\circ}$ ConDucts, we thought 

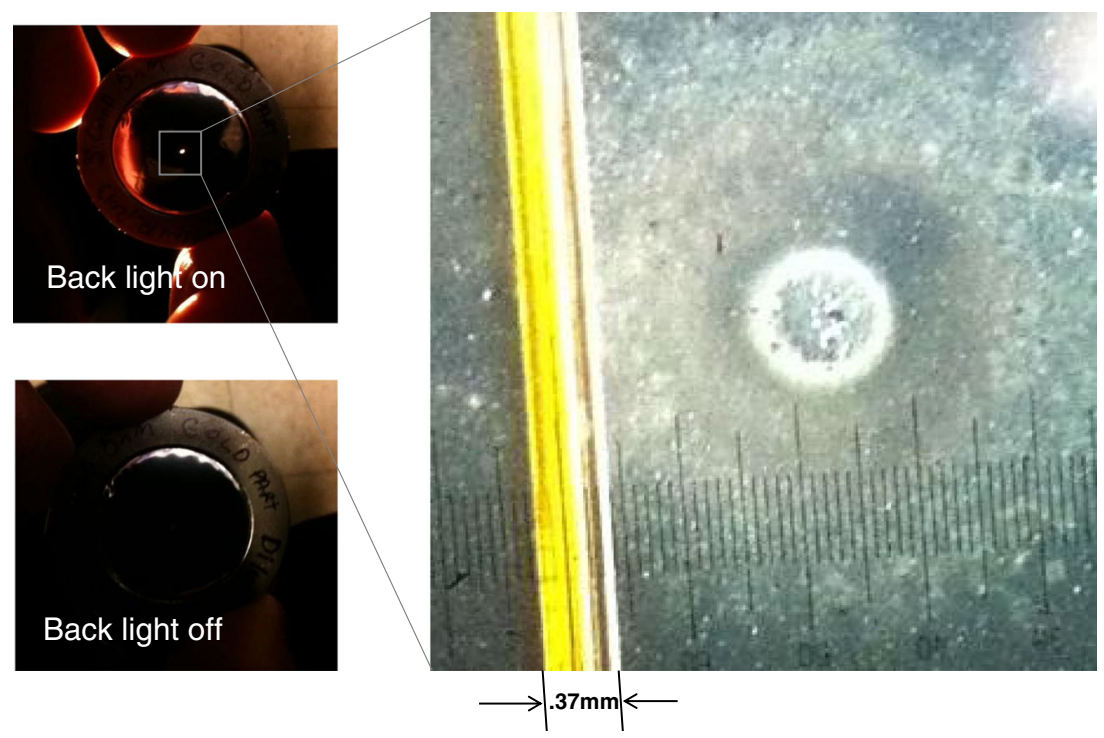

Figure 3. Hole ablated through a 1- $\mu$ m thick nickel foil placed in the path of a beam created by a $3^{\circ}$ ConDuct electrode. The distance between the exit of the inlet electrode and the target is $80 \mathrm{~mm}$. The beam contains $5 \mathrm{~nm}$ gold nanoparticles electrosprayed towards the ConDuct inlet. The inset shows a crater surrounding a tiny ablated hole in the foil. The diameter of the crater is $0.3-$ $0.4 \mathrm{~mm}$, which can be estimated from the image of the piece of quartz capillary (yellow) ( $0.37 \mathrm{~mm}$ diameter).

that these interfaces might prove to be relatively inefficient for heat transfer in this range [22,23]. Thus, to maximize efficiency of heat transfer from the electrode to the beam, we decided to use a low divergence $\left(2^{\circ}\right)$ ConDuct as the interface inlet. Figure $5 \mathrm{a}$ shows a schematic of a modified LCQ DECA XP mass spectrometer equipped with two atmosphere-to-vacuum ion interfaces: (i) the Thermo S-lens interface on the left-hand side and (ii) the new ConDuct interface on the right-hand side. This instrument can operate with both interfaces simultaneously, enabling us to compare their relative performances [21].
Figure S1 (Supplementary Information) shows spectra of a $100 \mathrm{fmol} / \mu \mathrm{L}$ solution of labeled and unlabeled peptides electrosprayed sequentially at the inlet of these two interfaces. The heaters of the S-lens interface metal capillary and the tube of the ConDuct interface were both set to $180^{\circ} \mathrm{C}$. All spectra were acquired for $1 \mathrm{~min}$. Although the LCQ ion trap fill-in time was set to the absolute minimum of the instrument $(0.2 \mathrm{~ms})$, the spectra of the peptides introduced through the ConDuct interface exhibited clear signs of trap overfilling, causing spacecharge effects that included mass shifts and broadening of the
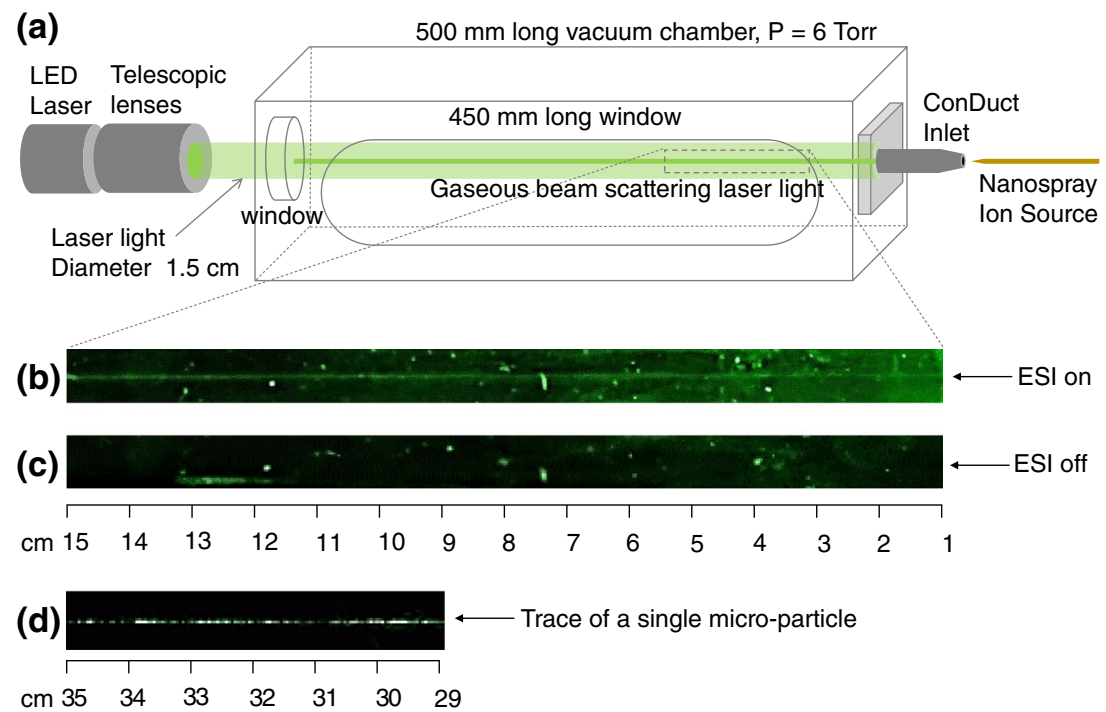

Figure 4. Visualization of the narrow, highly focused beam created by a ConDuct electrode transmitting ion species produced by an ESI source. (a) Schematic of the experimental setup for visualizing the ESI-produced beam. (b) A $15 \mathrm{~cm}$ region of the beam scattering light from a widely expanded laser beam propagating towards it. (c) Control image that shows no scattering from the beam when the ESI source is switched off. (d) Light scattering by a single microparticle interacting with a laser beam modulated at $200 \mathrm{kHz}$. Analysis of the image produced an estimate of the velocity of the microparticle of $(320 \pm 40) \mathrm{m} / \mathrm{s}$. 
(a)

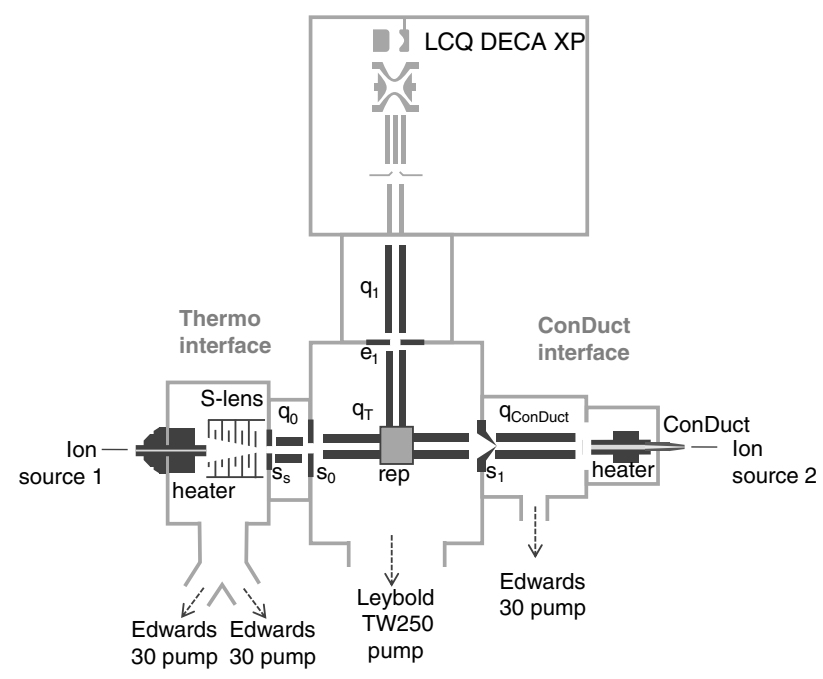

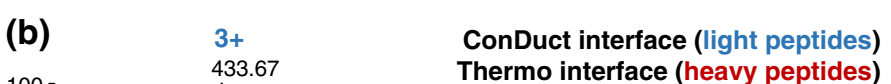
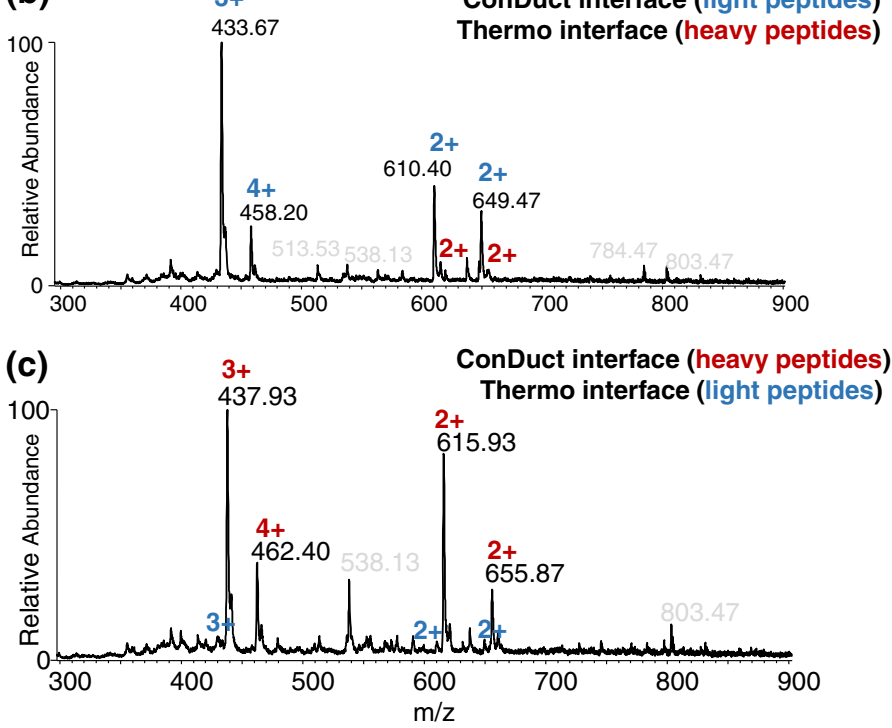

Figure 5. (a) Modified LCQ DECA XP mass spectrometer equipped with two atmosphere-to-vacuum ion interfaces: Thermo S-lens interface (left-hand side) and $2^{\circ}$ ConDuct interface (right-hand side). (b) Spectrum of unlabeled and labeled peptides electrosprayed simultaneously from two identical ESI sources positioned at two separate atmosphere-to-vacuum interfaces. The unlabeled peptides were electrosprayed through the ConDuct interface whereas labeled peptides were electrosprayed through the S-lens interface. (c) The nanospray ion sources were swapped and the measurements repeated.

(a)

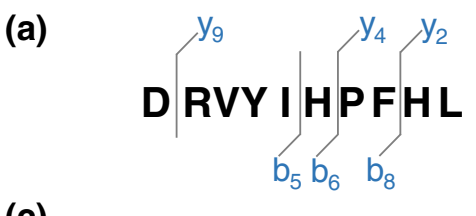

(b)

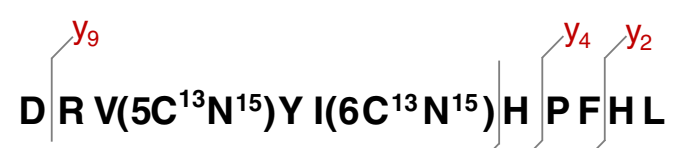

\section{(c)}
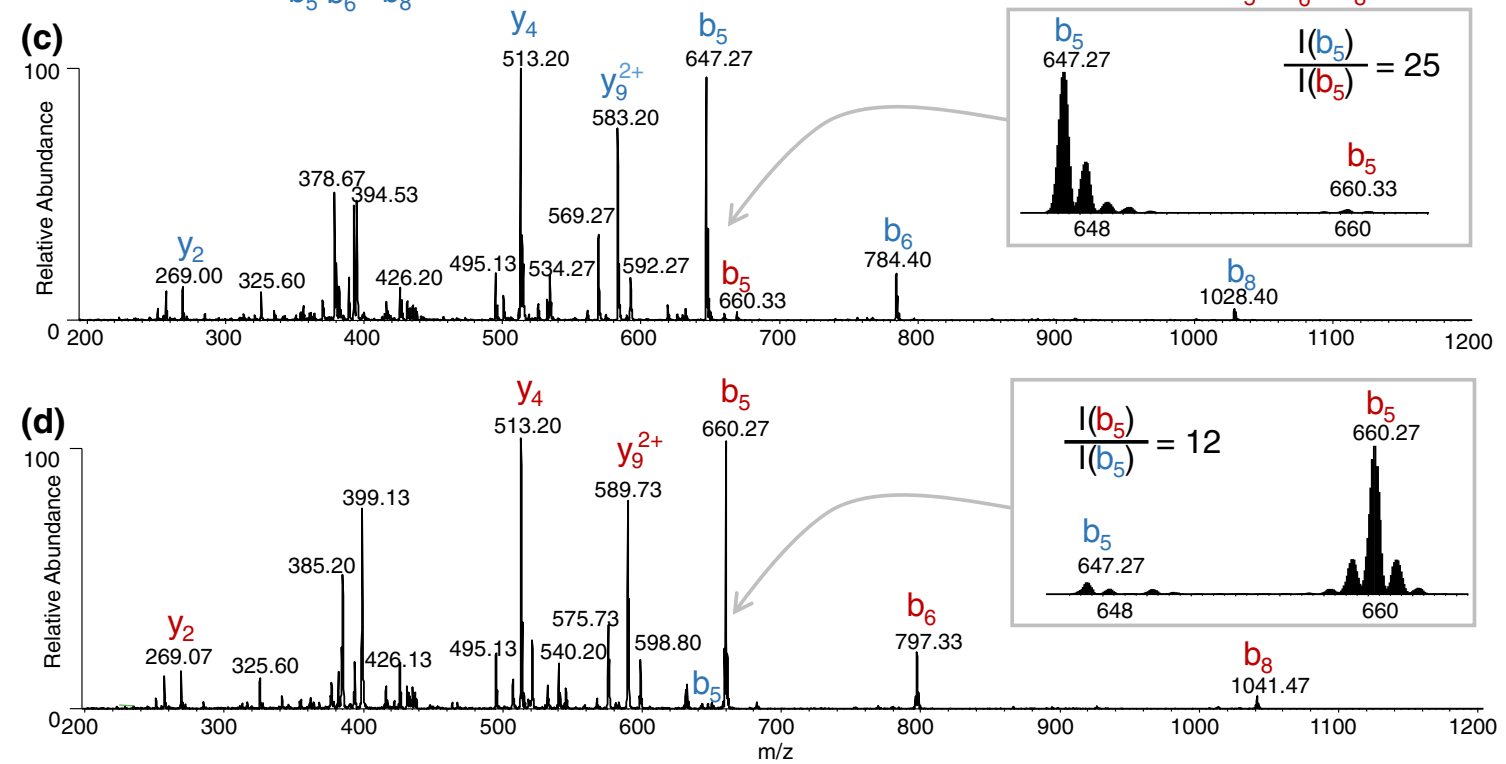

Figure 6. Fragmentation of angiotensin I peptides. (a) Unlabeled $\left(\mathrm{MM}_{\mathrm{avg}}=1296.5 \mathrm{u}\right)$ and (b) labeled with heavy isotopes $\left(\mathrm{MM}_{\mathrm{avg}}=\right.$ $1309.5 \mathrm{u}$ ). (c) MS/MS spectrum of the triply charged ion species selected at $\mathrm{m} / z(435 \pm 4)$ Th in the precursor spectrum (Figs. $5 \mathrm{~b}$ and c). The unlabeled (light) peptides were introduced through the ConDuct interface and the labeled (heavy) peptides were introduced through the Thermo interface. After the spectrum was collected, the electrospray ion sources were swapped and the data were collected again. (d) MS/MS spectrum of the triply charged ion species selected at $m / z(435 \pm 4)$ Th in the precursor spectrum. Unlabeled peptides were transmitted through the commercial (Thermo) interface and the labeled ones through the ConDuct interface. From the intensity ratios of the $b_{5}$ fragments of the unlabeled and labeled peptides in the two spectra, we calculated the ion transmission of the ConDuct interface was 17 times higher than that of the commercial interface. 
peaks. For both interfaces, the optimal signal-to-noise ratio in the spectra was observed when the tip of the nanospray emitter was 4-8 $\mathrm{mm}$ from the inlet and 1-2 $\mathrm{mm}$ off-center.

Figures $5 \mathrm{~b}$ and $\mathrm{c}$ show the spectra of a $100 \mathrm{fmol} / \mu \mathrm{L}$ solution of unlabeled and labeled peptides electrosprayed simultaneously at the inlets of the two atmosphere-to-vacuum interfaces. The mixture of unlabeled (light) peptides was first electrosprayed at the inlet of the ConDuct interface, whereas the mixture of the heavylabeled peptides was electrosprayed at the inlet of the S-lens interface (Figure $5 b$ ). Then, the nanospray ion sources were quickly swapped and the measurements repeated (Figure 5c). From the ratio of the intensities of the labeled (heavy) and unlabeled (light) peptide peaks, we deduced that the ConDuct interface transmitted significantly more ions than the S-lens interface.

Because it proved challenging to measure the relative transmission efficiency from the MS spectra alone - e.g., tailing of the intense ion peaks of the unlabeled peptides frequently masked the tiny peaks of the labeled ones - we decided to determine the intensity ratios from MS/MS spectra instead, which provided a higher effective dynamic range. To do this, we acquired MS/MS spectra of the triply charged precursor ions selected within a $\mathrm{m} / \mathrm{z}$ window of $(435 \pm 4)$ Th. Figure 6a shows one such fragmentation spectrum of the triply charged ions of the light- and heavy-labeled peptides. The inset represents the zoomed-in $\mathrm{m} / \mathrm{z}$ region 645-665, showing two peaks that correspond to the $b_{5}$ fragments of the unlabeled and labeled peptides. The ratio of the intensities of these peaks was 25 . To control for possible "asymmetry" of the two ion sources, we quickly swapped them and repeated the measurements. Figure $6 \mathrm{~b}$ shows the fragmentation spectrum and the inset zoomed into the vicinity of the $b_{5}$ fragments. The ratio of the intensities of the labeled to unlabeled peptide peaks, in this case, was 12 . We calculated the relative transmission efficiencies of both interfaces from the intensity ratios of the singly charged $b_{5}$ fragments of the unlabeled and labeled peptides in both spectra. Overall, the ion transmission of the ConDuct interface was 17 times higher (from the geometric mean $\sqrt{25 \times 12}=17)$ than the ion transmission of the commercial interface. We should emphasize that this is the best result we obtained so far, indicating the maximum ratio observed under the conditions and settings of DC and radio frequency (RF) voltages that allowed us to obtain somewhat comparable signal-to-noise spectra. However, the transmission efficiency through the S-lens interface could be moderately increased by increasing the RF amplitude of the $q_{0}$ quadrupole ion guide (Figure 5a and [21]). Although this can reduce the transmission efficiency advantage of the ConDuct interface from 17 to 6 (Figure S2, Supplementary Information), it causes considerable deterioration of the signal-tonoise ratios of the ions arriving via the commercial interface (Figure S3, Supplementary Information).

\section{Conclusions}

The unique properties of the ion beams produced by the ConDuct electrodes described here could prove to be invaluable for a variety of scientific applications. For MS research,
ConDucts have great potential as inlet electrodes in atmosphere-to-vacuum interfaces because of their ion transmission efficiency of close to $100 \%$. Our first ConDuct interface, which utilized plastic pipette tip electrodes, performed 400 times more efficiently than the original LCQ long metal capillary interface, and 2-3 times better than the newer S-lens interface used in certain modern commercial instruments [21]. In the present work, we showed that the ion transmission can be further increased by a factor of 6-17 by implementing a 2-degree metal ConDuct electrode in the interface. This improvement in transmission efficiency was achieved without significantly increasing the gas conductance of the interface.

In addition to the large gain in transmission efficiency, the ConDuct interface also provides improved signal-to-noise mass spectra through factors that we do not yet fully understand. We speculate that these factors could be connected to any or all of the processes of ionization, sampling, desolvation, and ion particle focusing in the vacuum. It is noteworthy that the best signal-to-noise spectra were obtained when the tip of the nanospray source was at a distance of 4 to $8 \mathrm{~mm}$ from the inlet of the ConDuct electrode. Such long distances likely ensure more complete evaporation of the microdroplets before they are sucked into the interface. This result correlated well with our observation of no scattering of laser light by the beam created by the ConDuct when the distance between the tip of the nanospray emitter and the inlet electrode was increased to more than $4 \mathrm{~mm}$. However, the velocity of the suction wind that entrains the droplets and ions to guide them into the ConDuct drops at least 16-fold at a distance of 4 to $8 \mathrm{~mm}$ versus $1 \mathrm{~mm}$, where entrainment is almost $100 \%$ efficient, diminishing the effectiveness of ion sampling into the inlet. It might prove to be possible that longer, low divergence ConDuct electrodes would allow us to move the tip of the nanospray emitter closer to the inlet, ideally 1 to $2 \mathrm{~mm}$, and still provide efficient desolvation through the heated walls of the electrode. In the future, we plan to increase the length of the metal ConDuct electrodes from $24 \mathrm{~mm}$ used in this work to $60 \mathrm{~mm}$.

Production of highly focused beams could also be used for deposition, modification, and even cutting of some materials and surfaces. One exciting possible application is the production of atmosphere-to-vacuum interfaces that provide a trainlike delivery of molecules and clusters to an area where they can be effectively sampled and analyzed because of the small radial dimensions of the beam. One example of such an application is femtosecond X-ray crystallography of proteins and protein microcrystals [35] that currently rely on either liquid jets [35] or aerodynamic lens stacks [36]. Both of these systems have serious limitations, which we speculate might be addressable using ConDuct-formed beams.

\section{Acknowledgments}

The authors acknowledge support for this work by the National Institutes of Health (R21 AI096069 and U54 GM103511 to B.T.C.). The authors are grateful to Eloy Wouters and Jean- 
Jacques Dunyach from Thermo Fisher Scientific for technical support and helpful discussions.

\section{References}

1. Pauly, H.: Atom, molecule, and cluster beams. I. Basic theory, production, and detection of thermal energy beams. Springer series on Atomic, Optical and Plasma Physics, $1^{\text {st }}$ edition, vol. 28, pp. 344. Springer, Berlin, Germany (2000)

2. Kantrowitz, A., Grey, J.: A high intensity source for the molecular beam. Part I. Theor. Rev. Sci. Instrum. 22, 328 (1951)

3. Dole, M., Mack, L.L., Hines, R.L.: Molecular beams of macro-ions. J. Chem. Phys. 49(5), 2240-2249 (1968)

4. Yamashita, M., Fenn, J.B.: Electrospray ion source. Another variation on the free-jet theme. J. Phys. Chem. 88(20), 4451-4459 (1984)

5. Fomina, N.S., Kretinina, A.V., Masyukevich, S.V., Bulovich, S.V., Lapushkin, M.N., Gall, L.N., Gall, N.R.: Transport of ions and charged droplets from the atmospheric region into a gas dynamic interface. J. Anal. Chem. 68(13), 1151-1157 (2013)

6. Page, J.S., Kelly, R.T., Tang, K., Smith, R.D.: Ionization and transmission efficiency in an electrospray ionization-mass spectrometry interface. J. Am. Soc. Mass Spectrom. 18, 1582-1590 (2007)

7. Piseri, P., Podesta, A., Bardorini, E., Milani, P.: Production and characterization of highly intense and collimated cluster beams by internal focusing in supersonic expansions. Rev. Sci. Instrum. 72(5), 2261-2267 (2001)

8. Wilm, M.S., Mann, M.: Electrospray and Taylor-cone theory, Dole's beam of macromolecules at last? Int. J. Mass Spectrom. Ion Processes 136, 167-180 (1994)

9. Wahl, J.H., Goodlett, D.R., Udseth, H.R., Smith, R.D.: Use of smalldiameter capillaries for increasing peptide and protein detection sensitivity in capillary electrophoresis-mass spectrometry. Electrophoresis 14, 448457 (1993)

10. Marginean, I., Kelly, R.T., Prior, D.C., LaMarche, B.L., Tang, K., Smith, R.D.: Analytical characterization of the electrospray ion source in the nanoflow regime. Anal. Chem. 80, 6573-6579 (2008)

11. Valaskovic, G.A., Murphy III, J.P., Lee, M.S.: Automated orthogonal control system for electrospray ionization. J. Am. Soc. Mass Spectrom. 15, 1201-1215 (2004)

12. Liang, X.R., Xia, Y., McLuckey, S.A.: Alternately pulsed nanoelectrospray ionization/atmospheric pressure chemical ionization for ion/ion reactions in an electrodynamic ion trap. Anal. Chem. 78, 3208-3212 (2006)

13. Thomson A.B.., Chernushevich I.V., Loboda A.V.: Trapping and processing ions in radio frequency ion guides. In: March, R.E., Todd, J.F.J. (eds.) Practical aspects of trapped ion mass spectrometry, $1^{\text {st }}$ edition, Vol. 10, chapter IV, pp. 525-544. CRC Press, Boca Raton, USA (2010)

14. Kelly, R.T., Tolmachev, A.V., Page, J.S., Tang, K., Smith, R.D.: The ion funnel: theory, implementations, and applications. Mass Spectrom. Rev. 29(2), 294-312 (2010)

15. Thomson. A.B...: Driving high sensitivity in biomolecular MS. Gen. Eng. Technol. News 32(20). Available at: http://www.genengnews.com/genarticles/driving-high-sensitivity-in-biomolecular-ms/4603 (2012). Accessed 17 December 2014

16. Morrisette, L.E., Goldberg, T.J.: Turbulent-flow separation criteria for over-expanded supersonic nozzles. NASA Tech. Papers 1207, 1-37 (1978)

17. Bajic, S.: Electrospray and atmospheric pressure chemical ionization mass spectrometer and ion source. US5756994 A, December 14 (1995)
18. Katsu, N.: Ion transport device, ion analyzer, and analyzer using supersonic molecular jet process, Japan patent application 275299 , priority number 2008308952. Shimadzu Corp. (2009)

19. Wang, X., Kruis, F.E., McMurry, P.H.: Aerodynamic focusing of nanoparticles: I. Guidelines for designing aerodynamic lenses for nanoparticles. Aerosol Sci. Technol. 39, 611-623 (2005)

20. Wang, X., Gidwani, A., Girshick, S.L., McMurry, P.H.: Aerodynamic focusing of nanoparticles: II. Numerical simulation of particle motion through aerodynamic lenses. Aerosol Sci. Technol. 39, 624-636 (2005)

21. Krutchinsky, A.N., Padovan, J.C., Cohen, H., Chait, B.T.: Maximizing ion transmission into a mass spectrometer with a novel conduct electrospray interface. J. Am. Soc. Mass Spectrom. 26, (2015, this issue). doi:10.1007/ s13361-014-1062-1

22. Herbert, M.V., Herd, R.J.: Boundary-layer separation in supersonic propelling nozzles. Aeronautical Research Council reports and memoranda. Report and memorada no.3421, pp. 71. Ministry of Aviation (HMSO), London, England, UK (1966)

23. Papamoschou, D., Zill, A.: Fundamental investigation of supersonic nozzle flow separation. AIAA-2004-1111. AIAA $42^{\text {nd }}$ Aerospace Sciences Meeting, Reno, NV, January 5-8 (2004)

24. Chowdhury, S.K., Katta, V., Chait, B.T.: An electrospray-ionization mass spectrometer with new features. Rapid Commun. Mass Spectrom. 4, 81-87 (1990)

25. Fenn, J.B., Mann, M., Meng, C.K., Wong, S.F., Whitehouse, G.M.: Electrospray ionization for mass spectrometry of large biomolecules. Science 246, 64-71 (1989)

26. Franzen, J.: Method and device for transport of ions in gas through a capillary. US patent 5736740 A (1995)

27. Sparrow, E.M., Abraham, J.P., Minkowycz, W.J.: Flow separation in a diverging conical duct: effect of Reynolds number and divergence angle. Int. J. Heat Mass Transf 52, 3079-3083 (2009)

28. Available at: http://en.wikipedia.org/wiki/Electrical_discharge_machining. Accessed 17 December 2014

29. Available at: http://en.wikipedia.org/wiki/Fibonacci_number. Accessed 17 December 2014

30. Chernushevich, I.V.; Loboda, A.V.; Thomson, B.A.; Krutchinsky, A.N.: Mass spectrometer multiple device interface for parallel configuration of multiple devices. US patent 7358488 B2, April 15 (2008)

31. Mueller, D., Brachthaeuser, Y., Derpmann, V., Klopotowsli, S., Langer, M., Polaczek, C., Kersten, H., Wissdorf, W., Benter, T.: Investigation of gas and ion-dynamics in heated glass and metal inlet capillaries. Work in Progress, Poster TP 732, ASMS Conference (2014)

32. Sanna, G., Tomassetti, G. In: Introduction to molecular beams gas dynamics, Chapter VIII, The supersonic free jet, pp. 251-278. Imperial College Press (2005)

33. Available at: https://www.lifetechnologies.com/order/catalog/product/ S12000. Accessed 17 December 2014

34. Available at: http://imagej.nih.gov/ij. Accessed 17 December 2014

35. Spence, J.C.H., Weierstall, U., Chapman, H.N.: X-ray lasers for structural and dynamic biology. Rep. Prog. Phys. 75, 1-25 (2012)

36. Bogan, M.J., Boutet, S., Chapman, H.N., Marchesini, S., Barty, A., Benner, H.W., Rohner, R., Frank, M., Hau-Riege, S.P., Bajt, S., Woods, B., Seibert, M.M., Iwan, B., Timneanu, N., Hajdu, J., Schulz, J.: Aerosol imaging with a soft X-ray free electron laser. Aerosol Sci. Technol. 44, i-vi (2010) 